\title{
ANTIBACTERIAL ACTIVITY OF Piper betle EXTRACTS ON Helicobacter pylori AND IDENTIFICATION OF POTENTIAL COMPOUNDS
}

\author{
Nguyen Thi Huyen Trang ${ }^{1, *}$, Do Thi Thanh Trung ${ }^{2 *}$, Nguyen Thi Thanh Thi ${ }^{2}$, Pham Thi \\ Luong Hang ${ }^{2,3}$, Pham Thi Vinh Hoa ${ }^{1}$, Pham Bao Yen ${ }^{3, * *}$ \\ ${ }^{1}$ Laboratory Center, Hanoi University of Public Health, Ha Noi, Vietnam \\ ${ }^{2}$ Faculty of Biology, VNU University of Science, Ha Noi, Vietnam \\ ${ }^{3}$ The Key Laboratory of Enzyme and Protein Technology (KLEPT), VNU University of \\ Science, Ha Noi, Vietnam
}

Received 14 June 2019, accepted 5 October 2019

\begin{abstract}
Helicobacter pylori is one of the most common infectious bacteria in the world that causes gastric diseases leading to cancer. The increase of multiple antibiotic resistance rates of $H$. pylori have been reported worldwide. Thus, development of novel drugs is urgently required. Piper betle has many therapeutic values in traditional medicine. In this study, therefore, we investigated antibacterial activity of $P$. betle extracts and their fractions against a $H$. pylori strain isolated in Vietnam. The agar disk diffusion assay showed inhibition zone of ethyl acetate extract and methanol extract from $P$. betle leaf that of were $46 \mathrm{~mm}$ and $32 \mathrm{~mm}$ in diameter, respectively. After fractionation of the ethyl acetate extract through silica gel column chromatography, two peaks, PD2 and PD3, out of 12 fractions showed the strongest antibacterial activity. PD2 was sub-fractionated further by re-chromatography on the silica gel column, and subfraction TK12 gave best resolution on LC-MS analysis. Finally, 4 potential compounds, quercetrin, calodenin B, vitexin and plicatipyrone, were identified in TK12 fraction.
\end{abstract}

Keywords: Helicobacter pylori, Piper betle, medicinal plants, gastric disease, compound.

Citation: Nguyen Thi Huyen Trang, Do Thi Thanh Trung, Nguyen Thi Thanh Thi, Pham Thi Luong Hang, Pham Thi Vinh Hoa, Pham Bao Yen, 2019. Antibacterial activity of Piper betle extracts on Helicobacter pylori and identification of potential compounds. Academia Journal of Biology, 41(4): 69-74. https://doi.org/10.15625/26159023/v41n4.13876.

*These authors are co-first authors

**Corresponding author email: yenpb@vnu.edu.vn

(O2019 Vietnam Academy of Science and Technology (VAST) 


\section{INTRODUCTION}

H. pylori is one of the most popular pathogens worldwide. According to the United States Center for Disease Control, approximately a half of the world's population is infected with $H$. pylori. Recent research showed that $H$. pylori infection rate is still high in most countries with variances among geographical regions and different age groups (Frenck et al., 2003). The prevalence of $H$. pylori infection in developing countries such as South America, Eastern Europe, and Asia is higher than in developed countries such as North Europe and North America. In some coutries in Africa, more than $90 \%$ of adults are identified positive for $\mathrm{H}$. pylori (Eusebi et al., 2014). According to the statistics, in Vietnam, roughly $60-70 \%$ of the population (about 56 millions) were infected with this bacterium (Fock \& Ang, 2010). Helicobacter pylori infection has been treated with combination of several antibiotics such as amoxicillin, tetracycline, clarithromycin and metronidazole. However, according to the statistics, the antibiotic resistance of $H$. pylori is increasing with presence of more multidrug-resistant strains (Binh et al., 2013). Thus, development of new drugs is an urgent demand.

It should be noted that Vietnam has a rich medicinal resource background for the screening of natural compounds possessing antibacterial ability against $H$. pylori. Among those medicinal plants, Piper betle $\mathrm{L}$ in the Piperaceae family has many therapeutic values including wound healing and antioxidant properties. $P$. betle leaf has been used for the treatment of various diseases like bad breath, boils and abscesses, conjunctivitis, constipation, headache, itches, mastitis, mastoiditis, leucorrhoea, otorrhoea, swelling of gum, rheumatism, cuts and injuries. The leaf has significant antimicrobial and antifungal activities against many pathogens (Ali et al., 2010; Agarwal et al., 2012). The Indian traditional medicine identified $P$. betle leaves has digestive and pancreatic lipase stimulant activities (Prabhu et al., 1995). Besides, $P$. betle leaf can inhibit progression of gastric ulceration, helping in the faster healing (Arawwawala et al., 2014). Thus, in this study, we aimed to examine antibacterial activity against $H$. pylori and identify potential compounds to develop anti-H. pylori drugs from the $P$. betle leaf.

\section{MATERIALS AND METHODS}

\section{Preparation of $\boldsymbol{P}$. betle leave extracts}

Piper betle leaves were collected, then dried at $40{ }^{\circ} \mathrm{C}$ and stored at $4{ }^{\circ} \mathrm{C}$. The leaves were extracted in ethyl acetate and methanol solvents following the procedure described in a previous study (Pham et al., 2017). Firstly, the powdered leaf part $(5 \mathrm{~g})$ was homogenized in $100 \mathrm{~mL}$ ethyl acetate using a mortar and pestle, followed by sonication for $5 \mathrm{~min}$ and stirring for $60 \mathrm{~min}$ at room temperature. The homogenate was centrifuged at $5,000 \times g$ for $10 \mathrm{~min}$ at $10^{\circ} \mathrm{C}$ and the supernatant was collected by filtration. The residue was extracted once again with $100 \mathrm{~mL}$ ethyl acetate and all supernatants were combined to obtain the ethyl acetate extract. Then the dried residue was successively extracted twice with $100 \mathrm{~mL}$ methanol with the same procedure to get a methanol extract. The organic solvents were removed by vacuum rotary evaporation. The dried extracts were weighed and stored at $-20^{\circ} \mathrm{C}$ until use.

\section{Isolation of Helicobacter pylori strains and cultivation}

Biopsies were stained with Giemsa agents to specify the presence of $H$. pylori bacteria. Positive samples were crushed by pestle blades and implanted in 5\% sheep blood and selective antibiotics for Helicobacter pylori selective supply. Agar plates were incubated at $37^{\circ} \mathrm{C}$ under an aerobic condition created by the GasPakTM environmental bag (Becton, Dickinson and Company, USA). Results were read after 7 days with the appearance of small, transparent colonies with diameter of 1$2 \mathrm{~mm}$. Tests used to identify H. pylori bacteria including Gram staining, the ability to produce urease, catalase, oxidase, and PCR with $H$. pylori -specific lipase primers. 


\section{Column chromatography}

In the first step, $25 \mathrm{~g}$ of silica gel was saturated in the first solvent system $(50 \% \mathrm{n}$ hexane: $50 \%$ ethyl acetate) and was packed into a column. The ethyl acetate extract (200 $\mathrm{mg}$ ) was applied on the top of silica gel. The components of extract were continuously eluted by six solvent systems with increasing polarity including 50\% n-hexane: 50\% ethyl acetate, $30 \%$ n-hexane: $70 \%$ ethyl acetate, $90 \%$ ethyl acetate: $10 \%$ methanol, $60 \%$ ethyl acetate: $40 \%$ methanol, $30 \%$ ethyl acetate: $70 \%$ methanol, and $100 \%$ methanol. The eluents were collected in tubes ( $3 \mathrm{ml} / \mathrm{tube}$ ) as a serial 12 fractions designted from PD1 to PD12.

In the second step, the PD2 fraction was further fractionated by subsequent silicagel column chromatography. An amount of 85 $\mathrm{mg}$ of PD2 fraction was loaded for onto the column and continuously eluted by six other solvent systems including $100 \%$ n-hexane, 70\% n-hexane: $30 \%$ ethyl acetate, $50 \%$ nhexane: $50 \%$ ethyl acetate, $30 \%$ n-hexane: $70 \%$ ethyl acetate, $100 \%$ ethyl acetate, and $50 \%$ ethyl acetate: 50\% methanol. The eluents were collected in tubes $(3 \mathrm{ml} / \mathrm{tube})$ as a serial 12 sub-fractions designated from TK1 to TK12.

\section{TLC chromatogram of 12 sub-fractions}

In this experiment, silica gel 60 F254 TLC Sheets $(6.5 \mathrm{~cm} \times 10 \mathrm{~cm})$ were used as a stationary phase. An amount of $0.5 \mathrm{mg}$ of each sub-fraction (TK1-TK12) was loaded on TLC. The TLC was then devoloped in solvent systems of $60 \%$ n- hexane: $40 \%$ ethyl acetate as mobile phase. Patterns on the TLC chromatogram were detected at $254 \mathrm{~nm}, 356$ nm UV light (UVP Lamp, Cambridge, UK).

\section{Liquid chromatography-mass spectrometry (LC-MS)}

High performance liquid chromatography (HPLC) coupled with mass spectrum was used to identify potential compounds in the active fraction. An amount of $3 \mu \mathrm{l}$ samples was injected into C18 - RP column (4.6 $\times$
$250 \mathrm{~mm}$, Phenomenex, USA) and separated in a gradient of $78-92 \% \mathrm{MeOH}$ in deionized water for $37 \mathrm{~min}$. The measurements were carried out at the Institute of Pharmaceutical Biology, University of Greifswald, Germany.

\section{Determination of antibacterial activity}

Agar diffusion method was performed as per Mitscher et al. (1972) with slight modifications (Mitscher et al., 1972). The dried extract was dissolved in DMSO to obtain a solution of $200 \mathrm{mg} / \mathrm{ml}$. Prior to the test, $20 \mu \mathrm{l}$ of extract $(200 \mathrm{mg} / \mathrm{ml})$ was added to the paper disc and air-dried for $3 \mathrm{hr}$. Helicobactor pylori was mixed into a suspension of the McFarland turbidity of 2 $\left(\mathrm{OD}_{625 \mathrm{~nm}}=0.451\right.$, approximately $6 \times 10^{8}$ $\mathrm{CFU} / \mathrm{ml}$ ) and streaked on a surface of blood agar plate. After that, the paper disc was placed on the surface of bacteria and incubated under a microaerophilic condition at $37^{\circ} \mathrm{C}$. Antibacterial zones were measured 5 days later.

\section{RESULTS AND DISCUSSION}

\section{Antibacterial activity of the extracts}

The ethyl acetate extract from Piper betel leaves has 1.5 times stronger anti-bacterial activity against $H$. pylori compared to the methanol extract (46 vs. $32 \mathrm{~mm}$ inhibition zone diameter, respectively). This result is comparable with the previous publication of the inhibitory effect of $P$. betle leaf extract against H. pylori (Nguyen Van Toai, 2003).

Therefore, the ethyl acetate extract of the $P$. betle leaf was selected for further isolation of active compounds using chromatography method.

\section{Antibacterial activity of silica-gel column chromatography fractions}

After silica-gel column chrpmatograpy of the ethyl acetate extract, 9/12 fractions had antibacterial activity against $H$. pylori with diameter $\geq 10 \mathrm{~mm}$ (figure 1 ).

In detail, 12 fractions can be divided into 4 groups based on $H$. pylori inhibitory activity by the diameter of inhibition zone (IZD): no activity (PD1); the weak group with IZD $<10$ 
mm (PD6 and 7 fraction); moderate group with IZD range of 10-30 mm (PD 4, 5, 8-12); the strong group with IZD fluctuating > 30 $\mathrm{mm}$ (PD2, 3). Apparently the antibacterial activity against $H$. pylori is localized mainly in fraction PD2 followed by PD3. Thus, PD2 fraction was analyzed further to find antibacterial compounds against $H$. pylori.
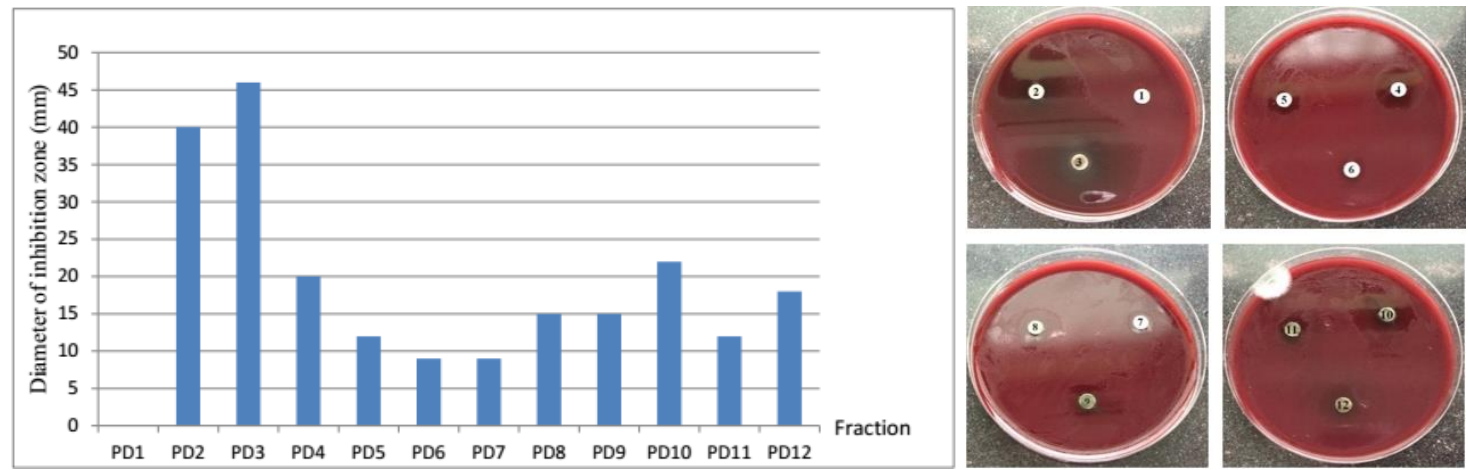

Figure 1. Antibacterial activity of 12 fractions against $H$. pylori

Identification of potential antibacterial compound in Piper betle leaf

\section{Sub-fractionation of PD2 fraction}

Before LC-MS analysis, PD2 fraction was fractionated further into sub-fractions by silica gel column chromatography. From
$85 \mathrm{mg}$ of PD2 fraction, 12 sub-fractions (TK1-TK12) were collected after elution with 6 solvent systems. TLC analysis (figure 2) revealed that 12 sub-fractions were different from each other with distinct TLC patterns. All of these sub-fractions were analysed using LC-MS.

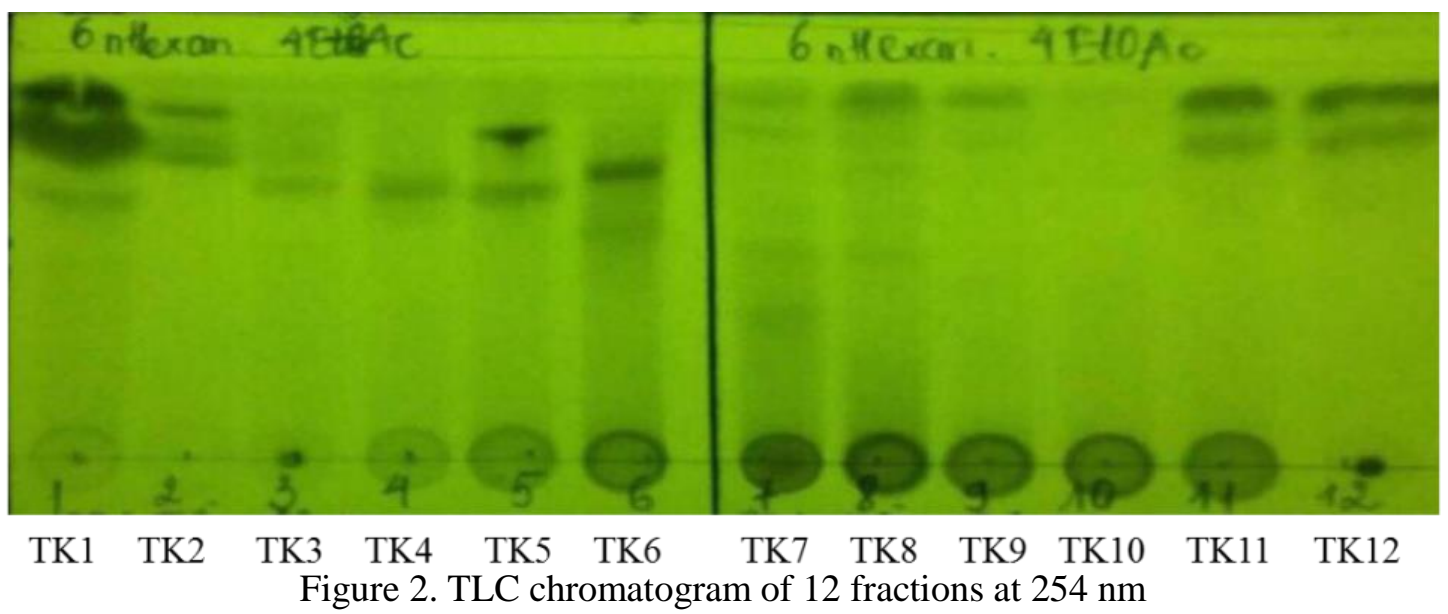

Identification the potential compounds in TK12 fraction

All of 12 sub-fractions (TK1-TK12) were analyzed by analytical HPLC with detection at $190 \mathrm{~nm}$ in $37 \mathrm{~min}$. The chromatograms of 12 sub-fractions were overlayed and shown in Figure 3a. Among 12 fractions, TK12 fraction gave the best spectrophotometric signal of its components. There were 8 peaks eluated at the corresponding time of minute $2,5,12.5$, 13.2, 15.5, 18.5, 20.5 and 23 (Figure 3a), which were analyzed on MS. MS of TK12 fraction at $13.2 \mathrm{~min}$ indicated the occurrence of 5 pairs of compounds with $16 \mathrm{Da}$ intervals in molecular mass. 

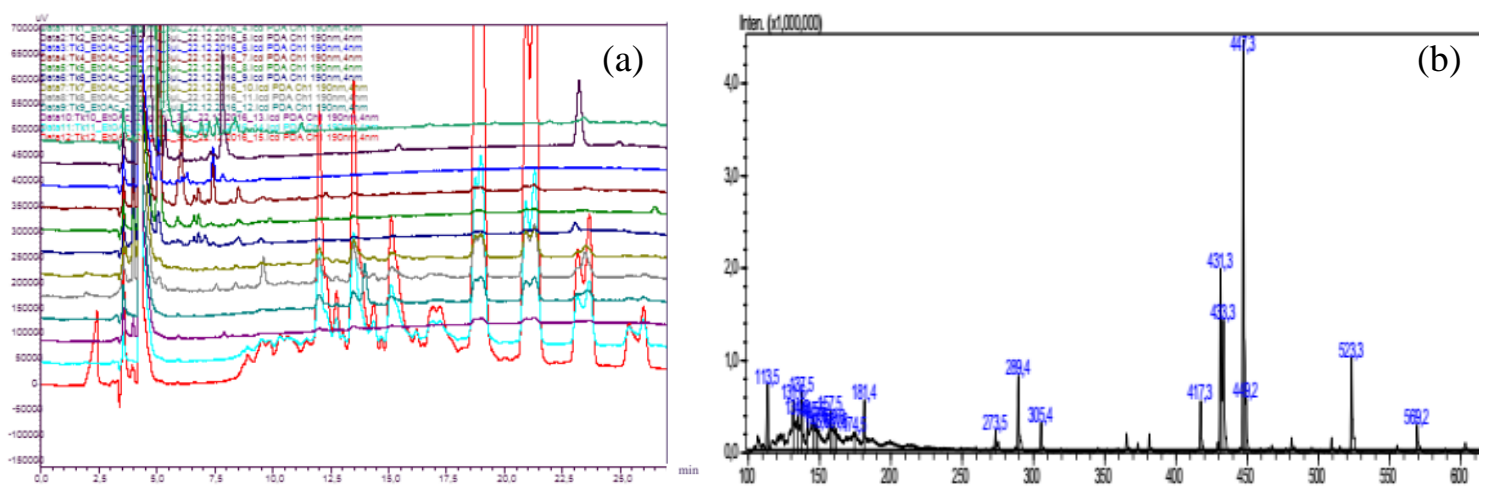

Figure 3. (a) Overlay HPLC chromatograms of 12 sub-fractions (TK1-TK12), (b) Mass spectrum of compounds appeared at $13.2 \mathrm{~min}$ in negative mode. Molecular weight of each peak is given as a blue fonts

We determined two potential compound groups including Group 1 with molecular weight ranging from 274.5-306.4 $\mathrm{Da}$ and Group 2 from 418.3-450.2 $\mathrm{Da}$. The compounds in each group were divided into pairs with $16 \mathrm{Da}$ apart such as Group 1
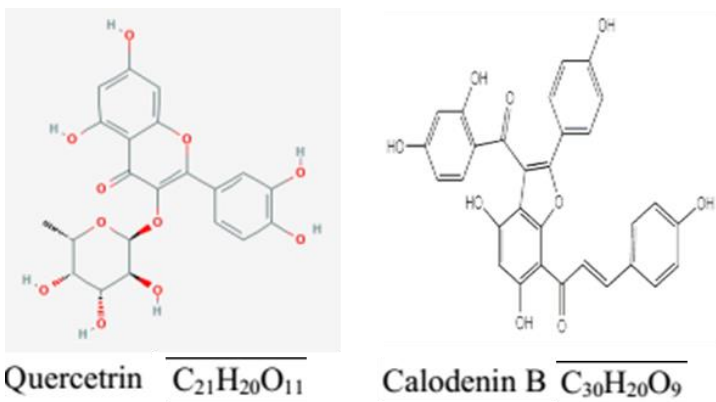

including 2 pairs: $274.5-290.4$ and $290.4-$ 306.4, Group 2 including 3 pairs: 418.3$434.3,432.3-448.3$ and 434.3-450.2. The investigation of compounds based on the library of natural products identified 4 compounds as shown in figure 4.
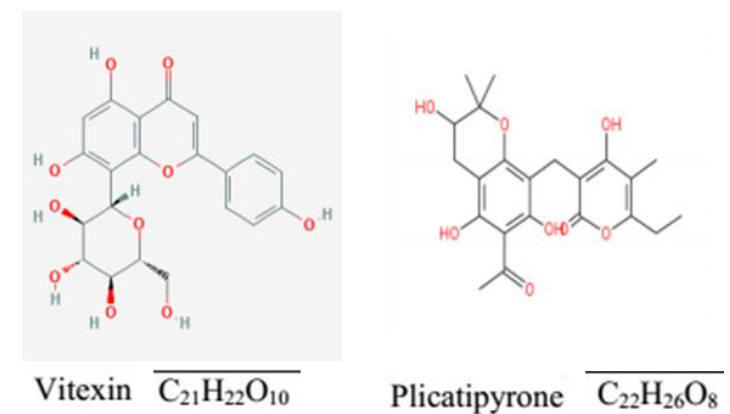

Figure 4. Prediction of potential compounds

These compounds, including quercetrin, MW of 448; calodenin, MW of 524; vitexin, MW of 434; plicatipyrone, MW of 418, were previously extracted from other plants with strong antibacterial activity, and also included in the list of compounds from $P$. betle leaf (Pradhan et al., 2013; Snow et al., 2016; Ghosh et al., 2014; Tang et al., 2003). Yet, none of those compounds has investigated their anti- $H$. pylori activity.

\section{CONCLUSION}

Ethyl acetate extract of $P$. betle leaf sample had strong anti-H. pylori activity with diameter of inhibition zone of $46 \mathrm{~mm}$. Two consecutive fractionations of this extract using silica gel column chromatography yielded 12 fractions and the anti- $H$. pylori activity was concentrated in PD2 fraction. By the second run of the column chromatography, 12 subfractions (TK1-TK12) from PD2 fraction with the first column. Among sub-fractions, TK12 gave the best resolution in the component analysis by LC-MS, in which 4 potential compounds have been predicted to be quercetrin, calodenin $\mathrm{B}$, vitexin and plicatipyrone.

Acknowledgements: This study received funding from the National Foundation for 
Science and Technology Development (NAFOSTED) with the code: 106-NN.022013.55. We kindly thank to Dr. Simon Merdivan (Institute of Pharmaceutical Biology, University of Greifswald, Germany) for LC-MS measurements. We thank the Key Laboratory of Enzyme and Protein Technology, VNU University of Science for providing research facilities.

\section{REFERENCES}

Agarwal A. K., Tripathi S. K., Xu T., Jacob M. R., Li X.-C., Clark A. M., 2012. Exploring the molecular basis of antifungal synergies using genome-wide approaches. Frontiersin Microbiology, 3: 115.

Ali I., F. G. Khan, K. A. Suri, B. D. Gupta, N. K. Satti, P. Dutt, F. Afrin, G. N. Qazi, I. A. Khan, 2010. In vitro antifungal activity of hydroxychavicol isolated from Piper betle L. Annals of clinical microbiology and antimicrobials, 9(1): 7 .

Arawwawala, L. D. A. M., Arambewela, L. S. R., Ratnasooriya, W. D., 2014. Gastroprotective effect of Piper betle Linn. leaves grown in Sri Lanka. Journal of Ayurveda and integrative medicine, 5(1): 38

Binh T. T., S. Shiota, L. T. Nguyen, D. D. Ho, H. H. Hoang, L. Ta, D. T. Trinh, T. Fujioka, Y. Yamaoka, 2013. The incidence of primary antibiotic resistance of Helicobacter pylori in Vietnam. Journal of clinical gastroenterology, 47(3): 233.

Chinou I. B., V. Roussis, D. Perdetzoglou, O. Tzakou, A. Loukis, 1997. Chemical and Antibacterial Studies of two Helichrysum Species of Greek Origin1. Planta medica, 63(02): 181-183.

Eusebi L. H., R. M. Zagari, F. Bazzoli, 2014. Epidemiology of Helicobacter pylori infection. Helicobacter, 19(s1): 1-5.

Fock K. M., T. L. Ang, 2010. Epidemiology of Helicobacter pylori infection and gastric cancer in Asia. Journal of gastroenterology and hepatology, 25(3): 479-486.
Frenck Jr, R. W., Clemens, J., 2003. Helicobacter in the developing world. Microbes and infection, 5(8): 705-713.

Ghosh R., K. Darin, P. Nath and P. Deb., 2014. An overview of various Piper species for their biological activities. International Journal of Pharmaceutical Sciences Review and Research, 3(1): 67-75.

Hang T. L. Pham, Lien T. T. Nguyen, Tuan A. Duong, Dung T. T Bui, Que T. Doan, Ha T. T. Nguyen, Sabine Mundt, 2017. Diversity and bioactivities of nostocacean cyanobacteria isolated from paddy soil in Vietnam. Systematic and Applied Microbiology, 40: 470-481.

Mitscher L. A., Leu R. P., Bathala M. S., Wu W. N., Beal J. L., 1972. Antimicrobial agents from higher plants-I: introduction, rationale and methodology. Lloydia, 35: 157-166.

Nguyen Van Toai, 2003. Initial study of antiHelicobacter pylori by total extract from Piper betle in in vitro and pre-clinical trial, $\mathrm{PhD}$ Thesis.

Prabhu M., K. Platel, G. Saraswathi, Srinivasan, K., 1995. Effect of orally administered betel leaf (Piper betle Linn.) on digestive enzymes of pancreas and intestinal mucosa and on bile production in rats. Indian journal of experimental biology, 33(10): 752-756.

Pradhan D., K. Suri, D. Pradhan, Biswasroy, P., 2013. Golden Heart of the Nature: Piper betle L. Journal of Pharmacognosy and Phytochemistry, 1(6): 147-167.

Snow Setzer M., J. Sharifi-Rad, Setzer, W. N., 2016. The Search for Herbal Antibiotics: An In-Silico Investigation of Antibacterial Phytochemicals. Antibiotics, 5(3): 30.

Tang S., P. Bremner, A. Kortenkamp, C. Schlage, A. I. Gray, S. Gibbons, M. Heinrich, M., 2003. Biflavonoids with cytotoxic and antibacterial activity from Ochna macrocalyx. Planta medica, 69(03): 247-253. 
